\title{
Optimal resource allocation for cognitive radio networks with primary user outage constraint
}

\author{
Peng Lan', Lizhen Chen ${ }^{1}$, Guowei Zhang ${ }^{2}$ and Fenggang Sun ${ }^{*}$
}

\begin{abstract}
In this paper, we investigate the problem of power allocation in cognitive underlay networks, where a secondary user (SU) is allowed to coexist with a primary user (PU). We consider three transmission models for the secondary link: (i) one-way transmission with relay assisted, (ii) two-way transmission with a direct link, and (iii) two-way transmission with relay assisted. In conventional interference-limited cognitive networks, the instantaneous channel state information (CSI) of a PU is required to suppress SU's transmit power to guarantee the quality of service (QoS) of the $\mathrm{PU}$, which increases the feedback burden in practice. To tackle this issue, in this article we take primary outage probability as a new criterion to measure the QoS of the PU, where only the statistical CSI of the PU is required. Firstly, we derive the primary outage constraints for the three models, respectively. Then, with the newly obtained constraints, we formulate optimization problems to maximize the channel rate of the SU. Finally, we derive the optimal solutions for power allocation with respect to different parameters, respectively. Simulation results verify the performance improvement of the proposed schemes.
\end{abstract}

Keywords: Cognitive radio, Cooperative communication, Power allocation, Quality of service, Two-way networks

\section{Introduction}

In cognitive underlay networks, a secondary user (SU) is allowed to share the spectrum with a primary user (PU) as long as the quality-of-service (QoS) requirement of the primary transmission is guaranteed [1]. The main advantage of cognitive underlay systems lies in its efficient utilization of radio spectrum, which makes it as a promising solution to tackle the spectrum scarcity problem [2]. However, the transmit power of the SU needs to be strictly controlled to satisfy PU's QoS, which consequently degrades SU's performance. To tackle this issue, power allocation and cooperative relaying techniques are considered as two potential ways to improve SU's performance.

Cooperative relaying has been widely studied to extend the coverage, enhance the reliability, as well as the capacity of wireless systems [3-5]. One-way relaying transmission often operates in a half-duplex mode that can provide more spatial diversity but suffers from a substantial loss

*Correspondence: sunfg@sdau.edu.cn

${ }^{1}$ College of Information Science and Engineering, Shandong Agricultural University, Daizong Road No.61, 271018 Tai'an, Shandong, China

Full list of author information is available at the end of the article in spectral efficiency as compared to direct transmission. Fortunately, two-way relaying transmission has been proposed to overcome the spectral efficiency loss [6-8]. Combining cooperative relaying and cognitive systems can provide a promising solution to improve SU's performance. In this article, we consider underlay cognitive relay networks involving three different transmission models for secondary transmission as shown in Fig. 1. In the one-way transmission model, a secondary transmitter (denoted as S1) transmits signals to a secondary receiver (denoted as S2) with the help of a secondary relay (SR). In the two-way transmission model, two secondary transceivers (denoted as S1 and S2, respectively) exchanges their signals either in a direct link or in two-way relaying link.

To protect the PU while optimizing the SU's performance, various power allocation (PA) strategies have been investigated for underlay cognitive relay networks $[9,10]$. Subject to average/peak interference power constraint for PU, an optimal PA strategy was proposed in [9] to achieve the ergodic capacity for SU in cognitive one-way relay networks. With power limit for SU being further considered,

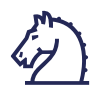

(C) 2015 Lan et al. Open Access This article is distributed under the terms of the Creative Commons Attribution 4.0 International License (http://creativecommons.org/licenses/by/4.0/), which permits unrestricted use, distribution, and reproduction in any medium, provided you give appropriate credit to the original author(s) and the source, provide a link to the Creative Commons license, and indicate if changes were made. 


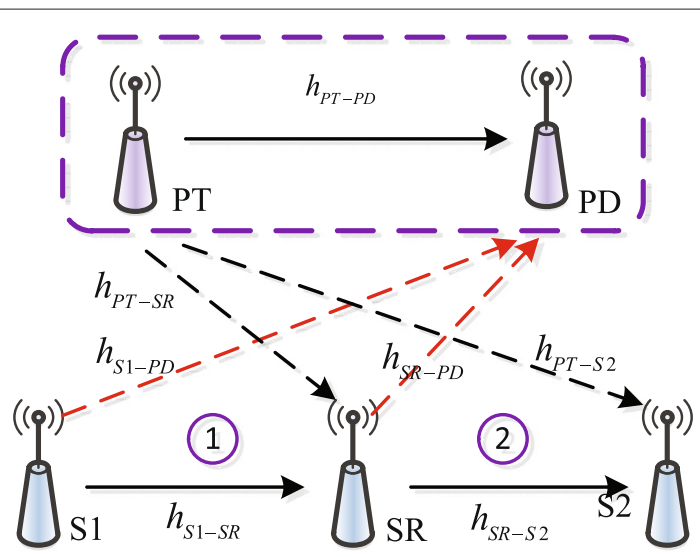

(a) one-way relaying model: $1^{\text {st }}$ phase in $\mathrm{S} 1 \rightarrow \mathrm{SR}$ transmission; $2^{\text {nd }}$ phase in $\mathrm{SR} \rightarrow \mathrm{S} 2$ transmission

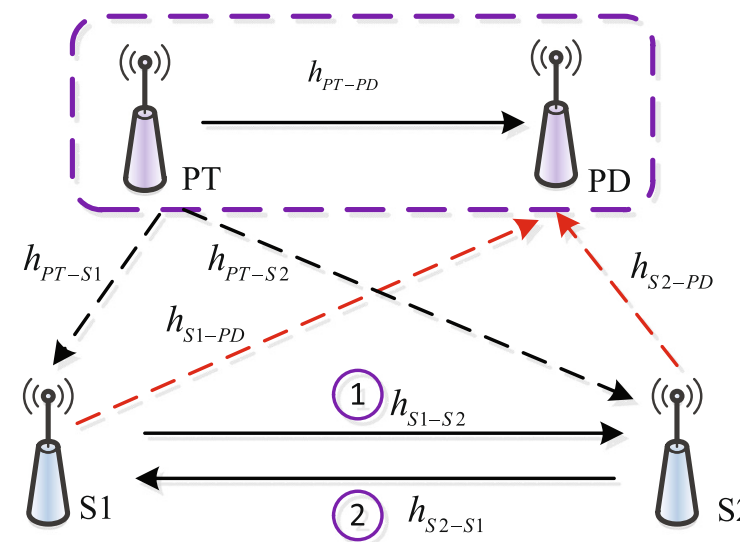

(b) two-way direct link model: $1^{\text {st }}$ phase in $\mathrm{S} 1 \rightarrow \mathrm{S} 2$ transmission; $2^{\text {nd }}$ phase in $\mathrm{S} 2 \rightarrow \mathrm{S} 1$

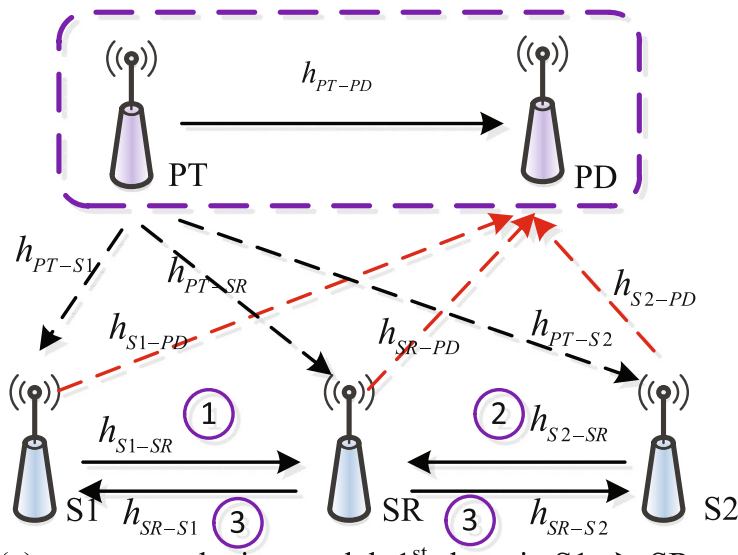

(c) two-way relaying model: $1^{\text {st }}$ phase in $\mathrm{S} 1 \rightarrow \mathrm{SR}$ transmission; $2^{\text {nd }}$ phase in S2 $\rightarrow$ SR transmission; $3^{\text {rd }}$ phase in $\mathrm{SR} \rightarrow \mathrm{S} 1, \mathrm{~S} 2$

Communication channel $\quad--\cdots+$ Interference channel

Fig. 1 System model of cognitive underlay networks with three secondary transmission modes. a One-way transmission with relay assisted (OWT-RA), b two-way transmission with a direct link (TWT-DL), and c two-way transmission with relay assisted (TWT-RA)

the authors in [11] proposed optimal PA schemes to maximize the ergodic/outage capacity of SU. In [12], PA schemes were proposed under the joint constraints of outage probability requirement for PU and average/peak transmit power limit for SU. With relay selection, the secondary transmission can be further enhanced. Joint PA and relay selection was investigated in [13] to maximize the system throughput with limited interference to PU. In [14], the transmit power limit for SU was also taken into consideration. For amplify-and-forward (AF) cognitive relay networks with multiple SUs, joint relay assignment and PA was proposed in [15]. In cognitive two-way net- works, closed-form solutions for optimal PA were derived in [16] under the joint peak interference constraint for PU. For cognitive two-way relaying networks, optimal PA and relay selection scheme were studied in [17], where a pair of secondary transceivers communicate with each other assisted by a set of two-way AF relays. Further, the problem of relay selection and PA for the two-way relaying cognitive radio networks was investigated in [10], where the relays select between the AF and decode-and-forward (DF) protocols to maximize SU's sum rate.

In the aforementioned works [9, 10], SU's transmit power is controlled to keep the interference of $\mathrm{PU}$ under 
a predefined limit. In such interference-limited cognitive networks, the instantaneous channel state information (CSI) of PU is required to be known at SU to protect PU. Since a secondary network is typically not coordinated with a primary network and no dedicated feedback channel is available from PU to SU, the instantaneous CSI of PU can hardly be obtained, and unreliable CSI results in violation of the interference constraint. To deal with this critical obstacle, Zou et al. investigated the problem of relay selection to maximize the received signalto-interference-noise ratio (SINR) at SU under a novel independent primary outage constraint for PU [18], in which only the statistical CSI of PU is required at SU. The work in [18] restrained the transmit powers of secondary transmitter and secondary relay under individual primary outage constraint for PU. In [19], the authors introduced a new cooperative transmission scheme for overlay cognitive radio in which the secondary network exploits the primary retransmissions without requiring global CSI. Further in [20], the primary outage constraint due to secondary transmitter and secondary relay was jointly considered for the first time, and a closed-form solution for optimal PA and relay selection was derived in DF cognitive relay networks.

In this article, we investigate the problem of power allocation to maximize the achievable rate of SU in cognitive networks. Three secondary transmission models are considered: (i) one-way transmission with relay assisted (OWT-RA), (ii) two-way transmission with a direct link (TWT-DL), and (iii) two-way transmission with relay assisted (TWT-RA). We first derive the joint primary outage constraints for the three models, respectively. With these constraints, optimization problems for power allocation are formulated and optimal solutions are derived. Simulation results are provided to verify the performance improvement of our proposed schemes compared with the equal resource allocation schemes.

To be more specific, the main contributions of this article are described as follows.

- We derived the joint primary outage constraints for three secondary transmission models, OWT-RA, TWT-DL, and TWT-RA, respectively. Compared to the traditional interference-limited constraint for PU, the key advantage is that only the statistical CSI of PU is required at $\mathrm{SU}$, which is more practical.

- We proposed power allocation schemes for OWT-RA, TWT-DL, and TWT-RA to maximize the achievable rate of $\mathrm{SU}$, respectively. In addition, we also proposed power allocation schemes to ensure fairness for TWT-DL and TWT-RA.

- For comparison, we provided corresponding low-complexity equal resource allocation (ERA) schemes for each model. Simulation results show that our proposed schemes outperform greatly the corresponding ERA scheme. The reason is that primary outage constraint due to secondary transmission is considered jointly in our proposed schemes, while it is considered individually in the ERA schemes.

The rest of this article is organized as follows. System model and the joint primary outage constraint of the three transmit models are, respectively, described in Sections 2 and 3. Then, the proposed power allocation schemes of different models are provided in Sections 4, 5, and 6, respectively. Simulation results are given in Section 7 . Section 8 concludes the article.

\section{System and channel model}

In this article, we consider underlay cognitive networks with three secondary transmission models as shown in Fig. 1, in which primary system and secondary system coexists simultaneously. In the primary system, a primary transmitter (PT) sends data to a primary destination (PD). The secondary system is a cooperative relay system which consists of a pair of transceivers (denoted as $S 1$ and $S 2$ ) and/or $M$ SRs, in which $\mathrm{SR}_{i}$ (for $i=1, \ldots, M$ ) denotes the $i$ th relay. The $M$ relays are considered to be in a cluster, so that they are assumed to be approximately at the same position. This assumption simplifies the analysis and can represent a number of practical scenarios [19, 21, 22]. Time division multiple access (TDMA)-based protocol is used for the secondary transmissions, and three different transmission models for the secondary system are considered in this article.

Model 1-one-way transmission with relay assisted (OWT-RA)

In the OWT-RA model as shown in Fig. 1a, $S 1$ is the transmit node and $S 2$ is the receive node. The secondary transmission between $S 1$ and $S 2$ is assisted by SRs (i.e. $S 1$ $\rightarrow \mathrm{SR} \rightarrow S 2$ ). The whole transmission process is divided into two phases equally. In the first phase, $S 1$ broadcasts its message and SRs receive. In the second phase, the best relay is selected to amplify the received signal and forward it to $S 2$.

Model 2-two-way transmission with a direct link (TWT-DL)

In the TWT-DL model, $S 1$ and $S 2$ transmit to each other without the assistance of SRs (i.e. $S 1 \leftrightarrow S 2$ ). The transmission procedure is shown in Fig. 1b. The whole secondary transmission process is divided into two phases equally, where $S 1$ transmits to $S 2$ in the first phase and $S 2$ transmits to $S 1$ in the second phase.

Model 3-two-way transmission with relay assisted (TWT-RA)

In TWT-RA model, $S 1$ and $S 2$ exchange information with the help of SRs (i.e. $S 1 \leftrightarrow \mathrm{SR} \leftrightarrow S 2$ ) when no direct 
link between $S 1$ and $S 2$ is available. The two-way secondary transmission is completed in three equal phases, as shown in Fig. 1c. $S 1$ and $S 2$ transmit to SRs in the first and second phases, respectively. In the third phase, a decode-and-forward (DF) protocol is considered, the best SR decodes the signals received from $S 1$ and $S 2$ and jointly encodes the signal through XOR operation and forwards the signal to $S 1$ and $S 2$.

In the primary transmission, assume that $\mathrm{PT}$ transmits signal $x_{P}\left(E\left(\left|x_{P}\right|^{2}\right)=1\right)$ to PD with fixed power $P_{P T}$ and a data rate $R_{P}$; in the meantime, $S 1$ and/or $S 2$ intends to reuse this time resource to transmit their signals $x_{S 1}$ and $x_{S 2}\left(E\left(\left|x_{S 1}\right|^{2}\right)=1\right.$ and/or $\left.E\left(\left|x_{S 2}\right|^{2}\right)=1\right)$ to each other with powers $P_{S 1}$ and $P_{S 2}$, respectively. The channels are invariant during the transmission phases. The channel gain between any transmitter $i \in\{S 1, S 2, \mathrm{SR}, \mathrm{PT}\}$ and any receiver $j \in\{S 1, S 2, \mathrm{SR}, \mathrm{PD}\}$ is denoted as $h_{i-j}$. Assuming all links are independent and identically distributed (i.i.d.), zero-mean Rayleigh flat fading channels with variance $1 / \sigma_{i-j}^{2}$, where $\sigma_{i-j}^{2}$ is defined as $\sigma_{i-j}^{2}=d_{i-j}^{\gamma}, d_{i-j}$ is the distance between transmitter $i$ and receiver $j$, and $\gamma$ is the path-loss exponent [19]. It is assumed that SU has the instantaneous CSI of the secondary transmission links and the links from PT to SU, which can be obtained by a pilot-aided channel estimation or CSI feedback $[14,23]$. Moreover, the two SUs are assumed to know the average CSI between themselves and PD and also the average CSI between PT and PD. The thermal noises of receivers $n_{j}(j \in\{S 1, S 2, \mathrm{SR}, \mathrm{PD}\})$ are modeled as additive white Gaussian noises (AWGN) with mean zero and variance $N_{0}$.

\section{The primary outage constraint}

In cognitive underlay networks, $\mathrm{SU}$ is allowed to share PU's spectrum on a condition that the QoS of the primary transmission is not affected. Therefore, the transmit power of SU must be allocated appropriately to satisfy the primary QoS requirement. Traditionally, SU's transmit power is limited by the peak/average interference constraint for PU $[9,10]$. Specifically, SU can access PU's licensed spectrum as long as the induced interference from SU to PU is below the threshold. In such an interference-limited network, the instantaneous CSI of the link from SU to PU is required. However, this CSI can hardly be obtained, since the secondary network is typically not coordinated with the primary network and no dedicated feedback channel is available from PU to SU.

To tackle this issue in this article, we use the primary outage probability as a metric to quantify the QoS of the primary transmission. Specifically, we consider that the primary outage probability should be kept below a predefined threshold PoutPri,Thr. The main advantage is that only the statistical CSI of the link from SU to PU is required, which is more practical. In the following, we derive the primary outage constraints for the three transmission models, OWT-RA, TWT-DL, and TWTRA, respectively.

According to the SINR at PD during the transmission phases, the conditional outage probability of $P U$ due to the transmission of node $n(n \in\{S 1, S 2, S R\})$ can be expressed as

$$
\text { Pout }(\mathrm{PU} \mid n)=\operatorname{Pr}\left(\log _{2}\left(1+r_{\mathrm{PD}}^{n}\right)<R_{P}\right),
$$

where $r_{\mathrm{PD}}^{n}=\frac{P_{\mathrm{PT}}\left|h_{\mathrm{PT}-\mathrm{PD}}\right|^{2}}{P_{n}\left|h_{n-\mathrm{PD}}\right|^{2}+N_{0}}=\frac{\widehat{P}_{\mathrm{PT}}\left|h_{\mathrm{PT}-\mathrm{PD}}\right|^{2}}{\widehat{P}_{n}\left|h_{n-\mathrm{PD}}\right|^{2}+1}(n \in\{S 1, S 2, \mathrm{SR}\})$ is the SINR at PD when node $n(n \in\{S 1, S 2, S R\})$ transmits signals. $\widehat{P}_{\mathrm{PT}}$ and $\widehat{P}_{n}$ are the equivalent transmit powers with normalized noise power, which are defined as $\widehat{P}_{\mathrm{PT}}=P_{\mathrm{PT}} / N_{0}$ and $\widehat{P}_{n}=P_{n} / N_{0}$, respectively. Note that $\left|h_{\mathrm{PT}-\mathrm{PD}}\right|^{2}$ and $\left|h_{n-\mathrm{PD}}\right|^{2}$ follow independent exponential distributions with parameters $1 / \sigma_{\mathrm{PT}-\mathrm{PD}}^{2}$ and $1 / \sigma_{n-\mathrm{PD}}^{2}$ respectively. Using the joint probability density function (PDF) of $\left|h_{\mathrm{PT}-\mathrm{PD}}\right|^{2}$ and $\left|h_{n-\mathrm{PD}}\right|^{2}$, the conditional outage probability (1) can be derived as

$$
\begin{aligned}
& \text { Pout }(\mathrm{PU} \mid n) \\
& =1-\frac{\widehat{P}_{\mathrm{PT}} \sigma_{\mathrm{PT}-\mathrm{PD}}^{2}}{\widehat{P}_{n} \sigma_{n-\mathrm{PD}}^{2}\left(2^{R_{P}}-1\right)+\widehat{P}_{\mathrm{PT}} \sigma_{\mathrm{PT}-\mathrm{PD}}^{2}} \exp \left(-\frac{2^{R_{P}}-1}{\widehat{P}_{\mathrm{PT}} \sigma_{\mathrm{PT}-\mathrm{PD}}^{2}}\right) .
\end{aligned}
$$

In order to derive the primary outage probability rout Pri , the probability of node $n$ transmits signals is required. Since TDMA protocol is used for secondary transmission as described in Section 2, therefore, the secondary transmission is divided into $k$ equal phases, where $k$ is decided by which transmission model is adopted (e.g., $k=2$ for the OWT-RA and TWT-DL models, $k=3$ for the TWTRA model). Let $P_{t}(n)$ represents the probability that the node $n$ transmits signals, we have $P_{\mathrm{t}}(n)=\frac{1}{k}$. Using the total probability formula, the primary outage probability can be expressed as

$$
\begin{aligned}
\text { Pout }_{\mathrm{Pri}} & =\sum_{n \in\{S 1, S 2, \mathrm{SR}\}} P_{t}(n) \text { Pout }(\mathrm{PU} \mid n) \\
& =\sum_{n \in\{S 1, S 2, \mathrm{SR}\}} \frac{1}{k} \text { Pout }(\mathrm{PU} \mid n),
\end{aligned}
$$

which should satisfy the constraint Pout Pri $\leq$ PoutPri,Thr.

3.1 The primary outage constraint for the OWT-RA model In the OWT-RA model, $S 1$ and SR transmit in two separate equal phases, i.e., $P_{t}(n)=\frac{1}{2}, n \in\{S 1$, SR $\}$. Assume the $i$ th relay is selected to forward, the primary outage can be denoted as

$$
\begin{aligned}
\text { Pout }_{\text {Pri }} & =P_{t}(\mathrm{~S} 1) \text { Pout }(\mathrm{PU} \mid S 1)+P_{t}\left(\mathrm{SR}_{i}\right) \text { Pout }\left(\mathrm{PU}_{\mid} \mathrm{SR}_{i}\right) \\
& =\frac{1}{2} \text { Pout }(\mathrm{PU} \mid S 1)+\frac{1}{2} \text { Pout }\left(\mathrm{PU} \mid \mathrm{SR}_{i}\right) \\
& \leq \text { PoutPri,Thr. }
\end{aligned}
$$


Substitute (2) into (4), the primary outage constraint can be derived as

$$
\frac{g}{\widehat{P}_{S 1_{(i)}} \lambda_{S 1}+g}+\frac{g}{\widehat{P}_{\mathrm{RS}_{i}} \lambda_{\mathrm{SR}_{i}+g}} \geq 2 \rho,
$$

where $\rho=\left(1-\right.$ Pout $\left._{\mathrm{Pr}} \mathrm{i}, \mathrm{Th} r\right) \exp \left(\frac{2^{R_{P}}-1}{\widehat{P}_{\mathrm{PT}} \sigma_{\mathrm{PT}-\mathrm{PD}}^{2}}\right), g=$ $\widehat{P}_{\mathrm{PT}} \sigma_{\mathrm{PT}-\mathrm{PD}}^{2}$, and $\lambda_{n}=\sigma_{n-\mathrm{PD}}^{2}\left(2^{R_{P}}-1\right), n \in\{S 1, \mathrm{SR}\} . \widehat{P}_{\mathrm{SR}_{i}}$ is the equivalent transmit power of the $i$ th relay, and $\widehat{P}_{S 1_{(i)}}$ is the equivalent transmit power of $S 1$ corresponding to the $i$ th relay.

For the underlay cognitive networks, the QoS of PU should be conservatively guaranteed, that is, PoutPri,Thr should take a small value or at least no larger than 0.5 [12]. Therefore, $\rho>0.5$ always holds. Moreover, since the upper bound of $\frac{g}{\widehat{P_{S 1_{(i)}} \lambda_{S 1}+g}}+\frac{g}{\widehat{P}_{\mathrm{RS}_{i}} \lambda_{\mathrm{SR}_{i}}+g}$ is 2 , the secondary transmission is enabled only when $\rho<1$. Otherwise, the powers $\widehat{P}_{S 1_{(i)}}$ and $\widehat{P}_{\mathrm{SR}_{i}}$ should be set to zero, and the secondary transmission is not available. Based on the above analysis, the constraint $0.5<\rho<1$ will always be satisfied for SU power allocation in this model.

\subsection{The primary outage constraint for the TWT-DL model}

In the TWT-DL model, the secondary transmission is divided into two equal phases, i.e., $P_{t}(n)=\frac{1}{2}, n \in$ $\{S 1, S 2\}$. The primary outage constraint can be denoted as

$$
\begin{aligned}
\text { Pout }_{\text {Pri }} & =P_{t}(S 1) \text { Pout }(\mathrm{PU} \mid S 1)+P_{t}(S 2) \text { Pout }(\mathrm{PU} \mid \\
& =\frac{1}{2} \text { Pout }(\mathrm{PU} \mid S 1)+\frac{1}{2} \text { Pout }(\mathrm{PU} \mid S 2) .
\end{aligned}
$$

With the similar analysis with the OWT-RA model, we derive the primary outage constraint as

$$
\frac{g}{\widehat{P}_{S 1} \lambda_{S 1}+g}+\frac{g}{\widehat{P}_{S 2} \lambda_{S 2}+g} \geq 2 \rho,
$$

where $g, \rho$, and $\lambda_{n}, n \in\{S 1, S 2\}$ follow the similar definition as in the OWT-RA model.

\subsection{The primary outage constraint for the TWT-RA model} In the TWT-RA model, the secondary transmission is completed in three equal phases, i.e., $P_{t}(n)=\frac{1}{3}, n \in$ $\{S 1, S 2, \mathrm{SR}\}$. Assume that the $i$ th relay is selected, the primary outage constraint can be expressed as

$$
\begin{aligned}
& \text { Pout }_{\text {Pri }} \\
& =P_{t}(S 1) \text { Pout }(\mathrm{PU} \mid S 1)+P_{t}(S 2) \text { Pout }(\mathrm{PU} \mid S 2) \\
& \quad+P_{t}\left(\mathrm{SR}_{i}\right) \text { Pout }\left(\mathrm{PU} \mid \mathrm{SR}_{i}\right) \\
& =\frac{1}{3} \text { Pout }(\mathrm{PU} \mid S 1)+\frac{1}{3} \text { Pout }(\mathrm{PU} \mid S 2)+\frac{1}{3} \text { Pout }\left(\mathrm{PU} \mid \mathrm{SR}_{i}\right) .
\end{aligned}
$$

With the similar analysis with the OWT-RA model, we derive the primary outage constraint as

$$
\frac{g}{\widehat{P}_{S 1_{(i)}} \lambda_{S 1}+g}+\frac{g}{\widehat{P}_{S 2_{(i)}} \lambda{ }_{S 2}+g}+\frac{g}{\widehat{P}_{\mathrm{SR}_{i}} \lambda_{\mathrm{SR}_{i}}+g} \geq 3 \rho,
$$

where $\widehat{P}_{S 1_{(i)}}$ and $\widehat{P}_{S 2_{(i)}}$ denote the equivalent transmit powers of $S 1$ and $S 2$ corresponding to the $i$ th relay.

\section{Power allocation for the OWT-RA model}

In this section, the problem of power allocation is studied to maximize the achievable rate of SU for the OWT-RA model.

The secondary transmission is divided into two phases, and the received signal at $\mathrm{SR}_{i}$ in the first phase can be expressed as

$$
y_{\mathrm{SR}_{i}}=\sqrt{P_{\mathrm{S}_{(i)}}} h_{S 1-\mathrm{SR}_{i}} x_{S}+\sqrt{P_{\mathrm{PT}}} h_{\mathrm{PT}-\mathrm{SR}_{i}} x_{P}+n_{\mathrm{SR}_{i}} .
$$

In the second phase, the signal received at $S 2$ can be expressed as

$$
y_{S 2}=\sqrt{P_{\mathrm{SR}_{i}}} G_{i} h_{\mathrm{SR}_{i}-S 2} y_{\mathrm{SR}_{i}}+\sqrt{P_{\mathrm{PT}}} h_{\mathrm{PT}-S 2} x_{P}+n_{S 2},
$$

where $G_{i}$ denotes the normalization gain of $\mathrm{SR}_{i}$

$$
G_{i}=\sqrt{\frac{1}{P_{S 1_{(i)}}\left|h_{S 1-\mathrm{SR}_{i}}\right|^{2}+P_{\mathrm{PT}}\left|h_{\mathrm{PT}-\mathrm{SR}_{i}}\right|^{2}+N_{0}}} .
$$

Based on (10), (11), and (12), the received SINR at $S 2$ after the two transmission phases is then given as

$$
r_{S 2}=\frac{G_{i}^{2} P_{S 1_{(i)}}\left|h_{S 1-\mathrm{SR}_{i}}\right|^{2} P_{\mathrm{SR}_{i}}\left|h_{\mathrm{SR}_{i}-S 2}\right|^{2}}{G_{i}^{2} P_{\mathrm{SR}_{i}}\left|h_{\mathrm{SR}_{i}-S 2}\right|^{2}\left(P_{\mathrm{PT}}\left|h_{\mathrm{PT}_{\mathrm{SR}}}\right|^{2}+N_{0}\right)+P_{\mathrm{PT}}\left|h_{\mathrm{PT}-S 2}\right|^{2}+N_{0}} .
$$

By defining $G_{S 1-\mathrm{SR}_{i}}=\frac{\left|h_{S 1-\mathrm{SR}_{i}}\right|^{2}}{\widehat{P}_{\mathrm{PT}}\left|h_{\mathrm{PT}-\mathrm{SR}_{i}}\right|^{2}+1}$ and $G_{\mathrm{SR}_{i}-S 2}=$ $\frac{\left|h_{\mathrm{SR}_{i}-S 2}\right|^{2}}{\widehat{P}_{\mathrm{PT}}\left|h_{\mathrm{PT}-S 2}\right|^{2}+1},(13)$ can be rewritten as

$$
r_{S 2}=\frac{\widehat{P}_{S 1_{(i)}} G_{S 1-\mathrm{SR}_{i}} \widehat{P}_{\mathrm{SR}_{i}} G_{\mathrm{SR}_{i}-S 2}}{\widehat{P}_{S 1_{(i)}} G_{S 1-\mathrm{SR}_{i}}+\widehat{P}_{\mathrm{SR}_{i}} G_{\mathrm{SR}_{i}-S 2}+1} .
$$

\subsection{Equal resource allocation}

As noted in Section 3, the equivalent powers $\widehat{P}_{S 1_{(i)}}$ and $\widehat{P}_{\mathrm{SR}_{i}}$ must satisfy the primary outage constraint (5). A simple but not optimal way to meet the constraint without coordination between $S 1$ and $\mathrm{SR}_{i}$ would be $\frac{g}{\bar{P}_{S 1_{(i)}} \lambda_{S 1}+g} \geq$ $\rho$ and $\frac{g}{\widehat{P}_{\mathrm{SR}_{i}} \lambda \mathrm{SR}_{i}+g} \geq \rho$ [18], and the equivalent transmit powers $\widehat{P}_{S 1_{(i)}}$ and $\widehat{P}_{\mathrm{SR}_{i}}$ should satisfy

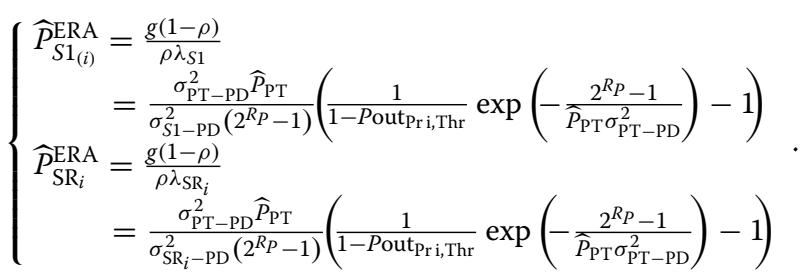

We denote this scheme as the equal resource allocation (ERA) scheme. From (15), the powers of $S 1$ and $\mathrm{SR}_{i}$ are dominantly determined by the QoS requirement of 
PU and the average channel gain of the links from PT to PD and from itself to PD. The static property allows the ERA scheme to allocate powers individually with low complexity. However, the only concern of the ERA scheme is to guarantee the primary transmission while the secondary transmission is ignored; therefore, it can not reach an optimal performance. Next, we will jointly allocate the transmit power between $S 1$ and $S R$ by taking into consideration the primary outage constraint (5).

\subsection{Optimal power allocation}

In the OWT-RA model, the SINR of $S 2$ is the benchmark to quantify the SU's performance; therefore, the aim of the power allocation is to maximize the SU's SINR as shown in (14). The optimization problem can be formulated as

$$
\left(\widehat{P}_{S 1_{(i)}}^{\mathrm{opt}}, \widehat{P}_{\mathrm{SR}_{i}}^{\mathrm{opt}}\right)=\arg \max _{\widehat{P}_{S 1_{(i)}}, \widehat{P}_{\mathrm{SR}_{i}}} \frac{\widehat{P}_{S 1_{(i)}} G_{S 1-\mathrm{SR}_{i}} \widehat{P}_{\mathrm{SR}_{i}} G_{\mathrm{SR}_{i}-S 2}-\mathrm{SR}_{i}+\widehat{P}_{\mathrm{SR}_{i}} G_{\mathrm{SR}_{i}-S 2}+1}{,}
$$

subject to

$$
\begin{aligned}
& \frac{g}{\widehat{P}_{S 1_{(i)}} \lambda_{S 1}+g}+\frac{g}{\widehat{P}_{\mathrm{SR}_{i}} \lambda_{\mathrm{SR}_{i}}+g} \geq 2 \rho, \\
& \widehat{P}_{S 1} \geq 0, \widehat{P}_{S 2} \geq 0 .
\end{aligned}
$$

This above problem is convex since its objective function (16) is concave and its constraint (17a) is convex and $(17 \mathrm{~b})$ is linear. Using the Lagrange multiplier method, the optimal power allocation problem of (16) and (17) can be given as

$$
\begin{aligned}
L\left(\widehat{P}_{S 1_{(i)}}, \widehat{P}_{\mathrm{SR}_{i}}, \lambda\right)= & \frac{1}{\widehat{P}_{S_{\mathcal{I}_{(i)}}} G_{S 1-S R_{i}}}+\frac{1}{\widehat{P}_{\mathrm{SR}_{i}} G_{S R_{i}-S 2}} \\
& +\frac{1}{\widehat{P}_{S 1_{(i)}} G_{S 1-S R_{i}} \widehat{P}_{\mathrm{SR}_{i}} G_{\mathrm{SR}_{i}-S 2}} \\
& +\lambda\left(\frac{g}{\widehat{P}_{S 1_{(i)}} \lambda_{S 1}+g}+\frac{g}{\widehat{P}_{\mathrm{SR}_{i}} \lambda \mathrm{SR}_{i}+g}-2 \rho\right),
\end{aligned}
$$

where $\lambda \geq 0$ represents the Lagrange multiplier. By applying the Karush-Kuhn-Tucker (KKT) optimality conditions [24], we can obtain

$$
\begin{aligned}
& \lambda\left(\frac{g}{\widehat{P}_{S 1_{(i)}} \lambda{ }_{S 1}+g}+\frac{g}{\widehat{P}_{\mathrm{SR}_{i}} \lambda \mathrm{SR}_{i}+g}-2 \rho\right)=0, \\
& \frac{\partial L}{\partial \widehat{P}_{S 1_{(i)}}}=0 \text { and } \frac{\partial L}{\partial \widehat{P}_{\mathrm{SR}_{i}}}=0 .
\end{aligned}
$$

The direct calculation of (19a) and (19b) yields the optimal solutions of the equivalent powers $\widehat{P}_{S 1_{(i)}}^{\text {opt }}$ and $\widehat{P}_{\mathrm{SR}_{i}}^{\text {opt }}$ as
From the derived solution (20), the optimal powers of $S 1$ and SR can be allocated. Different from the ERA scheme in (15), the powers of $S 1$ and $\mathrm{SR}_{i}$ are decided not only by the QoS requirement of PU and the average channel gain of the links from PT to PD and from itself to PD but also by the instantaneous channel gain of the secondary link. Moreover, the average channel gains of the interference links from $S 1$ and SR to PD are jointly considered in our schemes. To be more specific, if the average channel gain of one interference link is dominantly stronger that the another, without loss of generality, we assume $\lambda_{S 1}$ is larger than $\lambda_{\mathrm{SR}_{i}}$. According to (20), the proposed scheme will suppress the transmit power of $S 1$ but allocate more power to $\mathrm{SR}_{i}$ without violating the primary outage constraint. However, the ERA scheme only suppresses the power of $S 1$ when $\lambda_{S 1}$ is larger than $\lambda_{\mathrm{SR}_{i}}$. Therefore, the proposed scheme can reach a better trade-off between $S 1$ and SR than the ERA scheme, which can enhance the secondary transmission obviously.

It is noted that the power of $S 1$ varies with respect to different SRs. Since the secondary transmission is completed via relaying, the performance of the secondary link is dominantly affected by SR. In addition to power allocation, we can also enhance the secondary transmission by relay selection. To be more specific, once the optimal power allocation for all relays has been calculated, the relay with the highest SINR is selected as the best, i.e.,

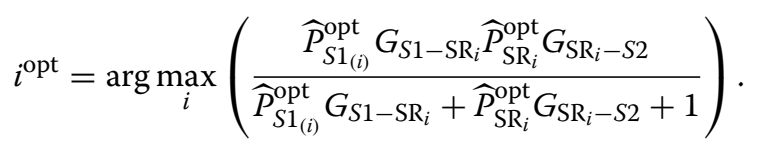

\section{Power allocation for the TWT-DL model}

In the cognitive TWT-DL network, since $S 1$ and $S 2$ are both transceivers and exchange information with each other, the achievable rate of $S 1$ and $S 2$ can be, respectively, defined as

$$
C_{S 1}=\frac{1}{2} \log _{2}\left(1+\alpha \widehat{P}_{S 1}\right) \text { and } C_{S 2}=\frac{1}{2} \log _{2}\left(1+\beta \widehat{P}_{S 2}\right),
$$

in which the two intermediate parameters of Eq. (22) are given as $\alpha=\frac{\left|h_{S 1-S 2}\right|^{2}}{\widehat{P}_{\mathrm{PT} T}\left|h_{\mathrm{PT}-S 2}\right|^{2}+1}$ and $\beta=\frac{\left|h_{S 1-S 2}\right|^{2}}{\widehat{P}_{\mathrm{PT}}\left|h_{\mathrm{PT}-S 1}\right|^{2}+1}$.

In the TWT-DL model, we enhance the secondary transmission by power allocation, and the two different goals are as follows: (1) to maximize the sum achievable

$$
\left\{\begin{array}{l}
\widehat{P}_{S 1_{(i)}}^{\mathrm{opt}}=\frac{2(1-\rho) g \sqrt{2(1-\rho) g G_{\mathrm{SR}_{i}-S 2}+(2 \rho-1) \lambda_{\mathrm{SR}_{i}}}}{(2 \rho-1) \lambda_{S 1} \sqrt{2(1-\rho) g G_{\mathrm{SR}_{i}-S 2}+(2 \rho-1) \lambda_{\mathrm{SR}_{i}}}+\sqrt{2(1-\rho) g \lambda_{S 1} \lambda_{\mathrm{SR}_{i}} G_{S 1-\mathrm{SR}_{i}}+(2 \rho-1) \lambda_{S 1}}} \\
\widehat{P}_{\mathrm{SR}_{i}}^{\mathrm{opt}}=\frac{2(1-\rho) g \sqrt{2(1-\rho) g G_{S 1-\mathrm{SR}_{i}}+(2 \rho-1) \lambda_{S 1}}}{(2 \rho-1) \lambda_{\mathrm{SR}_{i}} \sqrt{2(1-\rho) g G_{S 1-\mathrm{SR}_{i}}+(2 \rho-1) \lambda_{S 1}}+\sqrt{2(1-\rho) g \lambda_{S 1} \lambda_{\mathrm{SR}_{i}} G_{\mathrm{SR}_{i}-S 2}+(2 \rho-1) \lambda_{\mathrm{SR}_{i}}}}
\end{array}\right.
$$


rate and (2) to maximize the achievable rate of the weaker link which is referred as to guarantee fairness.

In the former goal, the sum achievable rate $C_{\text {Sum }}$ is denoted as

$$
C_{\text {Sum }}=C_{S 1}+C_{S 2}
$$

In the latter goal, to guarantee fairness, the achievable rate of $S 1$ and $S 2$ is expected to be the same. The sum achievable rate of $S 1$ and $S 2$ is decided by the weaker one. We denote the corresponding sum achievable rate for this case as $C_{\text {Fair }}$, which can be expressed as

$$
C_{\text {Fair }}=2 \min \left(C_{S 1}, C_{S 2}\right) \text {. }
$$

\subsection{Equal resource allocation}

Similar with the ERA scheme for the cognitive OWT-RA network as described in Section 4, the equivalent transmit powers $\widehat{P}_{S 1}$ and $\widehat{P}_{S 2}$ for the ERA scheme of the cognitive TWT-DL network should satisfy

$$
\left\{\begin{array}{rl}
\widehat{P}_{S 1}^{\mathrm{ERA}} & =\frac{g(1-\rho)}{\rho \lambda_{S 1}} \\
& =\frac{\sigma_{\mathrm{PT}-P D}^{2} \widehat{P}_{\mathrm{PT}}}{\left.\sigma_{S 1-P D}^{2} 2^{R_{P}}-1\right)}\left(\frac{1}{1-P_{\text {out }} \text { Pri, Thr }} \exp \left(-\frac{2^{R_{P}}-1}{\widehat{P}_{\mathrm{PT}} \sigma_{\mathrm{PT}-P D}^{2}}\right)-1\right) \\
\widehat{P}_{S 2}^{\mathrm{ERA}} & =\frac{g(1-\rho)}{\rho \lambda_{S 2}} \\
& =\frac{\sigma_{\mathrm{PT}-P D}^{2} \widehat{P}_{\mathrm{PT}}}{\sigma_{S 2-P D}^{2}\left(2^{R_{P}}-1\right)}\left(\frac{1}{1-P_{\text {out }} \text { Pri,Thr }} \exp \left(-\frac{2^{R_{P}}-1}{\widehat{P}_{\mathrm{PT}} \sigma_{\mathrm{PT}-P D}^{2}}\right)-1\right)
\end{array},\right.
$$

respectively. The ERA scheme in the TWT-DL also allocates powers individually. However, when considering the primary outage constraint, it is more meaningful to improve the SU's performance based on the joint primary outage constraint for $S 1$ and $S 2$. The SU performance desires further improvement through jointly allocating the transmission powers of $S 1$ and $S 2$.

\subsection{Optimal power allocation for data rate maximization (DRM)}

For the cognitive TWT-DL networks, the optimal equivalent power allocation to maximize the achievable rate of SU can be obtained by solving the following optimization problem

$$
\left(\widehat{P}_{S 1}^{\text {opt }}, \widehat{P}_{S 2}^{\text {opt }}\right)=\underset{\widehat{P}_{S 1}, \widehat{P}_{S 2}}{\arg C_{S u m}}
$$

subject to

$$
\begin{aligned}
& \frac{g}{\widehat{P}_{S 1} \lambda_{S 1}+g}+\frac{g}{\widehat{P}_{S 2} \lambda_{S 2}+g} \geq 2 \rho, \\
& \widehat{P}_{S 1} \geq 0, \widehat{P}_{S 2} \geq 0 .
\end{aligned}
$$

According to (22) and (23), the goal in (26) to maximize $C_{\text {Sum }}$ is equivalent to maximizing $\alpha \widehat{P}_{S 1}+\beta \widehat{P}_{S 2}+\alpha \beta \widehat{P}_{S 1} \widehat{P}_{S 2}$. For computation simplicity, by assuming $\widetilde{P}_{S 1}=\frac{\lambda_{S 1}}{g} \widehat{P}_{S 1}+1$,
$\widetilde{P}_{S 2}=\frac{\lambda_{S 2}}{g} \widehat{P}_{S 2}+1, \widetilde{\alpha}=\frac{\lambda_{S 2}}{\beta g}-1$, and $\widetilde{\beta}=\frac{\lambda_{S 1}}{\alpha g}-1$, the optimization problem can be further simplified as

$$
\begin{aligned}
\left(\widetilde{P}_{S 1}^{\text {opt }}, \widetilde{P}_{S 2}^{\text {opt }}\right) & =\arg \max _{\widetilde{P}_{S 1}, \widetilde{P}_{S 2}} g\left(\widetilde{P}_{S 1}, \widetilde{P}_{S 2}\right) \\
& =\widetilde{P}_{S 1} \widetilde{P}_{S 2}+\widetilde{\alpha} \widetilde{P}_{S 1}+\widetilde{\beta} \widetilde{P}_{S 2} .
\end{aligned}
$$

subject to

$$
\begin{aligned}
& \frac{1}{\widetilde{P}_{S 1}}+\frac{1}{\widetilde{P}_{S 2}} \geq 2 \rho, \\
& \widetilde{P}_{S 1} \geq 1, \widetilde{P}_{S 2} \geq 1 .
\end{aligned}
$$

Without considering the power limits (29b), to obtain the optimal solution, the constraint (29a) should satisfy with equality, which can be easily proved by contradiction. Take the equality $\frac{1}{\widetilde{P}_{S 1}}+\frac{1}{\widetilde{P}_{S 2}}=2 \rho$ into (28), the objective problem $g\left(\widetilde{P}_{S 1}, \widetilde{P}_{S 2}\right)$ can be further converted to a function with variable $\widetilde{P}_{S 1}$ as

$$
g\left(\widetilde{P}_{S 1}\right)=\left(\widetilde{\alpha}+\frac{1}{2 \rho}\right) \widetilde{P}_{S 1}+\frac{\left(\widetilde{\beta}+\frac{1}{2 \rho}\right)}{2 \rho\left(2 \rho \widetilde{P}_{S 1}-1\right)}+\frac{1}{2 \rho}\left(\widetilde{\beta}+\frac{1}{2 \rho}\right) .
$$

Take the partial derivative of $g\left(\widetilde{P}_{S 1}\right)$ with respect to $\widetilde{P}_{S 1}$, we can obtain

$$
\frac{d g\left(\widetilde{P}_{S 1}\right)}{d \widetilde{P}_{S 1}}=\widetilde{\alpha}+\frac{1}{2 \rho}-\frac{\widetilde{\beta}+\frac{1}{2 \rho}}{\left(2 \rho \widetilde{P}_{S 1}-1\right)^{2}}
$$

Take the limit $\widetilde{P}_{S 1} \geq 1$ into account, we can see that when $\frac{2 \widetilde{\beta} \rho+1}{2 \widetilde{\alpha} \rho+1}<(2 \rho-1)^{2}$, the partial derivative $\frac{d g\left(\widetilde{P}_{S 1}\right)}{d \widetilde{P}_{S 1}}>0$ and Eq. (30) is a monotonously increase function with $\widetilde{P}_{S 1}$. Therefore, $g\left(\widetilde{P}_{S 1}\right)$ can be maximized by maximizing $\widetilde{P}_{S 1}=\frac{1}{2 \rho-1}$ and $\widetilde{P}_{S 2}=1$, i.e., $\widehat{P}_{S 1}=\frac{2(1-\rho) g}{(2 \rho-1) \lambda_{S 1}}$ and $\widehat{P}_{S 2}=0$. In this case, the two-way transmission is retrograded as a one-way transmission, i.e., $S 1 \rightarrow S 2$. Similarly, if $\frac{2 \widetilde{\alpha} \rho+1}{2 \widetilde{\beta} \rho+1} \leq(2 \rho-1)^{2}$, the two-way transmission is retrograded as a one-way transmission from $S 2$ to $S 1$ with powers $\widehat{P}_{S 1}=0$ and $\widehat{P}_{S 2}=\frac{2(1-\rho) g}{(2 \rho-1) \lambda_{S 2}}$. With the above analysis, if $\min \left[\frac{2 \widetilde{\beta} \rho+1}{2 \widetilde{\alpha} \rho+1}, \frac{2 \widetilde{\alpha} \rho+1}{2 \widetilde{\beta} \rho+1}\right] \leq(2 \rho-1)^{2}$, the sum achievable rate can be maximized by $\max \left[\alpha \frac{2(1-\rho) g}{(2 \rho-1) \lambda_{S 1}}, \beta \frac{2(1-\rho) g}{(2 \rho-1) \lambda_{S 2}}\right]$. Specifically, if $\frac{\alpha}{\beta}>\frac{\lambda_{S 1}}{\lambda_{S 2}}$, the link of $S 1 \rightarrow S 2$ is enabled with powers $\left(\widehat{P}_{S 1}^{\text {opt }}, \widehat{P}_{S 2}^{\text {opt }}\right)=\left[\frac{2(1-\rho) g}{(2 \rho-1) \lambda_{S 1}}, 0\right]$. Otherwise, if $\frac{\alpha}{\beta} \leq \frac{\lambda_{S 1}}{\lambda_{S 2}}$, the link of $S 2 \rightarrow S 1$ is enabled with powers $\left(\widehat{P}_{S 1}^{\mathrm{opt}}, \widehat{P}_{S 2}^{\mathrm{opt}}\right)=\left[0, \frac{2(1-\rho) g}{(2 \rho-1) \lambda_{S 2}}\right]$.

For the case $\min \left[\frac{2 \widetilde{\beta} \rho+1}{2 \widetilde{\alpha} \rho+1}, \frac{2 \widetilde{\alpha} \rho+1}{2 \widetilde{\beta} \rho+1}\right]>(2 \rho-1)^{2}$, we cannot solve the optimization problem (28) with constraints (29a) and (29b) using the monotone property. Notice that the objective function is concave and the feasible region that meets every constraint in (28) forms a convex set. Thus, the solution to its Lagrange function also solves 
the maximization problem. The optimal power allocation problem (28) without considering the power limits (29b) can be formulated as

$L\left(\widetilde{P}_{S 1}, \widetilde{P}_{S 2}, \lambda\right)=\widetilde{P}_{S 1} \widetilde{P}_{S 2}+\widetilde{\alpha} \widetilde{P}_{S 1}+\widetilde{\beta}_{S 2}+\lambda\left(2 \rho-\frac{1}{\widetilde{P}_{S 1}}-\frac{1}{\widetilde{P}_{S 2}}\right)$,

where $\lambda \geq 0$ represents the Lagrange multiplier. By applying the KKT optimality conditions, we can obtain

$$
\begin{gathered}
\lambda\left(2 \rho-\frac{1}{\widetilde{P}_{S 1}}-\frac{1}{\widetilde{P}_{S 2}}\right)=0, \\
\frac{\partial L}{\partial \widetilde{P}_{S 1}}=0 \text { and } \frac{\partial L}{\partial \widetilde{P}_{S 2}}=0 .
\end{gathered}
$$

The direct calculation of (33a) and (33b) yields the optimal solutions

$$
\begin{aligned}
& \widetilde{P}_{S 1}^{\mathrm{opt}}=\frac{1}{2 \rho}\left(1+\sqrt{\frac{2 \widetilde{\beta} \rho+1}{2 \widetilde{\alpha} \rho+1}}\right), \\
& \widetilde{P}_{S 2}^{\mathrm{opt}}=\frac{1}{2 \rho}\left(1+\sqrt{\frac{2 \widetilde{\alpha} \rho+1}{\widetilde{\beta} \rho+1}}\right) .
\end{aligned}
$$

Integrated from the above analysis, the optimal solutions for the original problem (26) with constraints (27a) and (27b) can be written as

$$
\begin{aligned}
& \left(\widehat{P}_{S 1}^{\text {opt }}, \widehat{P}_{S 2}^{\text {opt }}\right) \\
& =\left\{\begin{array}{c}
\left(\frac{g}{\lambda_{S 1}}\left(\widetilde{P}_{S 1}^{\text {opt }}-1\right), \frac{g}{\lambda_{S 2}}\left(\widetilde{P}_{S 2}^{\text {opt }}-1\right)\right), \quad \min \left[\frac{2 \tilde{\beta} \rho+1}{2 \widetilde{\alpha} \rho+1}, \frac{2 \widetilde{\alpha} \rho+1}{2 \widetilde{\beta} \rho+1}\right]>(2 \rho-1)^{2} \\
\left(\frac{2(1-\rho) g}{(2 \rho-1) \lambda_{S 1}}, 0\right), \min \left[\frac{2 \tilde{\beta} \rho+1}{2 \widetilde{\alpha} \rho+1}, \frac{2 \widetilde{\alpha} \rho+1}{2 \widetilde{\beta} \rho+1}\right] \leq(2 \rho-1)^{2} \text { and } \frac{\alpha}{\beta}>\frac{\lambda_{S 1}}{\lambda_{S 2}} \\
\left(0, \frac{2(1-\rho) g}{(2 \rho-1) \lambda_{S 2}}\right), \min \left[\frac{2 \widetilde{\beta} \rho+1}{2 \widetilde{\alpha} \rho+1}, \frac{2 \widetilde{\alpha} \rho+1}{2 \tilde{\beta} \rho+1}\right] \leq(2 \rho-1)^{2} \text { and } \frac{\alpha}{\beta} \leq \frac{\lambda_{S 1}}{\lambda_{S 2}}
\end{array}\right.
\end{aligned}
$$

5.3 Optimal power allocation for data rate fairness (DRF) In the TWT-DL model, the transceivers $S 1$ and $S 2$ exchange information with each other. It is reasonable to consider fairness between the two transceivers. The problem of power allocation for SU with data rate fairness is formulated as

$$
\left(\widehat{P}_{S 1}^{\text {opt }}, \widehat{P}_{S 2}^{\text {opt }}\right)=\arg \max _{\widehat{P}_{S 1}, \widehat{P}_{S 2}} C_{\text {Fair }},
$$

with constraints (27a) and (27b).

To ensure fairness, the two transceivers should have the same rate, which is proved as follows. Assume that $P_{S 1}^{*}$ and $P_{S 2}^{*}$ reach the optimal solution in (36) and $C_{S 1} \neq C_{S 2}$. Without loss of generality, assume $C_{S 1}>C_{S 2}$. For this case, it is obvious that there must exist a smaller power $P_{S 1}^{\prime}$ satisfying $C_{S 1}=C_{S 2}$, which can obtain the same data rate of $\mathrm{SU}$, however, with less interference to PU and lower transmit power of SU. Therefore, the power allocation $\left(P_{S 1}^{\prime}, P_{S 2}^{*}\right)$ is superior to that of the allocation $\left(P_{S 1}^{*}\right.$, $P_{S 2}^{*}$ ), which means that to obtain the optimal solution,
$C_{S 1}=C_{S 2}$ should be satisfied, and the objective function (36) can be further rewritten as:

$$
\left(\widehat{P}_{S 1}^{\mathrm{opt}}, \widehat{P}_{S 2}^{\mathrm{opt}}\right)=\arg \max _{\widehat{P}_{S 1}, \widehat{P}_{S 2}}\left(C_{S 1}=C_{S 2}\right)
$$

To obtain the optimal solution, the constraint (27a) should satisfy with equality, which can be easily proved by contradiction. The optimal equivalent power allocation for this case should satisfy

$$
\widehat{P}_{S 1}^{\mathrm{opt}}=\gamma \widehat{P}_{S 2}^{\mathrm{opt}}
$$

where the parameter $\gamma$ is denoted as $\gamma=\frac{\beta}{\alpha}$. Thus, by substituting (38) into (27a), the optimal power can be calculated as

$$
\begin{aligned}
\widehat{P}_{S 2}^{\text {opt }}= & \frac{g\left(\gamma \lambda_{S 1}+\lambda_{S 2}\right)(1-2 \rho)}{4 \gamma \rho \lambda_{S 1} \lambda_{S 2}} \\
& +\frac{\sqrt{\left[g\left(\gamma \lambda_{S 1}+\lambda_{S 2}\right)(1-2 \rho)\right]^{2}+16 \gamma \lambda_{S 1} \lambda_{S 2} g^{2} \rho(1-\rho)}}{4 \gamma \rho \lambda_{S 1} \lambda_{S 2}}
\end{aligned}
$$

and $\widehat{P}_{S 1}^{\text {opt }}$ can be achieved as shown in (38).

\section{Power allocation for the TWT-RA model}

In the cognitive TWT-RA network, assume that the signal is severely attenuated between the two transceivers; thus, the direct link is not considered for transmission. The two-way transmission is completed with the help of secondary relays. The TDMA protocol is used, and the secondary transmission process is divided into three phases equally.

The received signals at the $i$ th SR during the first and second phases can be generally expressed as

$$
y_{\mathrm{SR}_{i}}=\sqrt{P_{\mathrm{Sm}_{(i)}}} h_{\mathrm{Sm}-\mathrm{SR}_{i}} x_{\mathrm{Sm}}+\sqrt{P_{\mathrm{PT}}} h_{\mathrm{PT}-\mathrm{SR}_{i}} x_{p}+n_{\mathrm{SR}_{i}},
$$

where $m=1$ when $S 1$ transmits signals and $m=2$ when $S 2$ transmits signals. Based on (40), the SINR at the $i$ th $S R$ in the first and second phases can be derived as

$$
r_{S m-\mathrm{SR}_{i}}=\frac{\left|h_{\mathrm{Sm}-\mathrm{SR}_{i}}\right|^{2}}{P_{\mathrm{PT}}\left|h_{\mathrm{PT}-\mathrm{SR}_{i}}\right|^{2}+N_{0}} P_{S_{(i)}} .
$$

In the third phase, after the cancelation of selfinterference, the received signal at $\operatorname{Sm},(m=1,2)$ corresponding to $i$ th SR can be denoted as

$$
y_{S m_{i}}=\sqrt{P_{S R_{i,(m)}}} h_{\mathrm{SR}_{i}-S m} x_{S m}+\sqrt{P_{\mathrm{PT}}} h_{\mathrm{PT}-S m} x_{p}+n_{S m},
$$


where $P_{S R_{i}}=\sum_{m=1}^{2} P_{S R_{i,(m)}}$ is the total transmit power of SR and $P_{\mathrm{SR}_{i,(m)}}$ is the transmit power of SR that allocates to $S_{m},(m=1,2)$. The SINR of $S_{m}$ can be expressed as

$$
r_{\mathrm{SR}_{i}-S m}=\frac{\left|h_{\mathrm{SR}_{i}-S m}\right|^{2}}{P_{\mathrm{PT}}\left|h_{\mathrm{PT}-S m}\right|^{2}+N_{0}} P_{S R_{i,(m)}} .
$$

Let $\alpha_{\mathrm{Sm}_{\mathrm{SR}}}=\frac{\left|h_{\mathrm{Sm}_{i}-\mathrm{SR}_{i}}\right|^{2}}{\widehat{P}_{\mathrm{PT}}\left|h_{\mathrm{PT}_{\mathrm{SR}}}\right|^{2}+1}$ and $\alpha_{\mathrm{SR}_{i}-\mathrm{Sn}}=$ $\frac{\left|h_{\mathrm{SR}_{i}-S n}\right|^{2}}{\widehat{P_{\mathrm{PT}}\left|h_{\mathrm{PT}-S n}\right|^{2}+1}}$ for $m, n=1,2$ and $m \neq n$. The achievable rate of $S 1$ is defined as

$$
\begin{aligned}
C_{S 1_{(i)}}= & \frac{1}{3} \min \left\{\log _{2}\left(1+\alpha_{S 1-\mathrm{SR}_{i}} \widehat{P}_{S 1_{(i)}}\right),\right. \\
& \left.\log _{2}\left(1+\alpha_{\mathrm{SR}_{i}-S 2} \widehat{P}_{S R_{i,(1)}}\right)\right\} .
\end{aligned}
$$

Similarly, we have the achievable rate for $S 2$ as

$$
\begin{aligned}
C_{S 2_{(i)}}= & \frac{1}{3} \min \left\{\log _{2}\left(1+\alpha_{S 2-\mathrm{SR}} \widehat{P}_{S 2_{(i)}}\right),\right. \\
& \left.\log _{2}\left(1+\alpha_{\mathrm{SR}_{i}-S 1} \widehat{P}_{S R_{i,(2)}}\right)\right\} .
\end{aligned}
$$

\subsection{Equal resource allocation}

With the similar analysis as the ERA schemes in the OWT-RA and TWT-DL networks, the ERA scheme in the TWT-RA network allocates powers of $\widehat{P}_{S 1}, \widehat{P}_{S 2}$, and $\widehat{P}_{S R}$ individually, which should satisfy the outage constraint

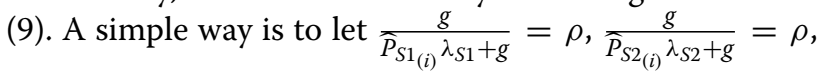
and $\frac{g}{\widehat{P}_{\mathrm{SR}_{i}} \lambda_{\mathrm{SR}_{i}}+g}=\rho$, and the equivalent transmit powers of the ERA scheme are $\widehat{P}_{S 1_{(i)}}^{\mathrm{ERA}}=\frac{g(1-\rho)}{\rho \lambda_{S 1}}, \widehat{P}_{S 2_{(i)}}^{\mathrm{ERA}}=\frac{g(1-\rho)}{\rho \lambda_{S 2}}$, and $\widehat{P}_{\mathrm{SR}_{i}}^{\mathrm{ERA}}=\frac{g(1-\rho)}{\rho \lambda_{\mathrm{SR}_{i}}}$ with $\widehat{P}_{S R_{i,(1)}}^{\mathrm{ERA}}=\widehat{P}_{S R_{i,(2)}}^{\mathrm{ERA}}=\frac{1}{2} \widehat{P}_{\mathrm{SR}_{i}}^{\mathrm{ERA}}$.

\subsection{Optimal power allocation scheme for data rate maximization (DRM)}

In this section, we consider the power allocation to maximize the sum achievable rate for the cognitive TWT-RA networks.

The sum achievable rate with respect to the $i$ th relay is given as

$$
C_{S i, \text { Sum }}=C_{S 1_{(i)}}+C_{S 2_{(i)}} .
$$

The optimal power allocation is to maximize the sum

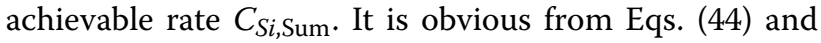
(45) that the achievable rate of $\operatorname{Sm}(m=1,2)$ is determined by the minimal value of the two-hop links: $S m \rightarrow$ $\mathrm{SR}_{i}$ and $\mathrm{SR}_{i} \rightarrow \mathrm{Sn}(m, n=1,2$ and $m \neq n)$. Therefore, the two links should achieve the same SINR, i.e., $\alpha_{S m-\mathrm{SR}_{i}} \widehat{P}_{S m_{(i)}}=\alpha_{\mathrm{SR}_{i}-S n} \widehat{P}_{S R_{i,(n)}}$. We have $\widehat{P}_{\mathrm{SR}_{i,(1)}}=$ $\beta_{1 i} \widehat{P}_{S 1_{(i)}}, \widehat{P}_{\mathrm{SR}_{i,(2)}}=\beta_{2 i} \widehat{P}_{S 2_{(i)}}$, and $\widehat{P}_{\mathrm{SR}_{i}}=\widehat{P}_{S R_{i,(1)}}+\widehat{P}_{\mathrm{SR}_{i,(2)}}=$ $\beta_{1 i} \widehat{P}_{S 1_{(i)}}+\beta_{2 i} \widehat{P}_{S 2_{(i)}}$, where $\beta_{1 i}=\frac{\alpha_{S 1-\mathrm{SR}_{i}}}{\alpha_{\mathrm{SR}_{i}-S 2}}$ and $\beta_{2 i}=\frac{\alpha_{S 2-S R_{i}}}{\alpha_{\mathrm{SR}_{i}-S 1}}$.
Based on the above analysis, the optimal power allocation can be allocated by solving the following optimization problem

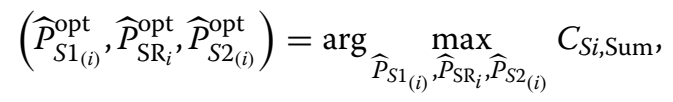

subject to

$$
\begin{aligned}
& \frac{g}{\widehat{P}_{S 1_{(i)}} \lambda_{S 1}+g}+\frac{g}{\widehat{P}_{S 2_{(i)}} \lambda_{S 2}+g}+\frac{g}{\widehat{P}_{\mathrm{SR}_{i}} \lambda_{\mathrm{SR}_{i}}+g} \geq 3 \rho, \\
& \widehat{P}_{\mathrm{SR}_{i}}=\beta_{1 i} \widehat{P}_{S 1_{(i)}}+\beta_{2 i} \widehat{P}_{S 2_{(i)}} .
\end{aligned}
$$

Using the Lagrange multiplier method, the optimal power allocation problem (47) with the constraints of (48a) and (48b) can be written as

$$
\begin{aligned}
L= & \sum_{m=1}^{2} \log _{2}\left(1+\alpha_{S m-\mathrm{SR}_{i}} \widehat{P}_{S_{m_{(i)}}}\right) \\
& +\lambda_{1}\left(\widehat{P}_{\mathrm{SR}_{i}}-\beta_{1 i} \widehat{P}_{S 1_{(i)}}-\beta_{2 i} \widehat{P}_{S 2_{(i)}}\right) \\
& +\lambda_{2}\left(\frac{g}{\widehat{P}_{S 1_{(i)}} \lambda \lambda_{S 1}+g}+\frac{g}{\widehat{P}_{S 2_{(i)}} \lambda{ }_{S 2}+g}\right. \\
& \left.+\frac{g}{\widehat{P}_{\mathrm{SR}_{i}} \lambda_{S R_{i}}+g}-3 \rho\right),
\end{aligned}
$$

where $\lambda_{1}$ and $\lambda_{2}$ represent nonnegative dual variables.

The Lagrange dual function can be obtained by

$$
\widehat{P}_{S 1_{(i)}} \max _{\widehat{P}_{S 2_{(i)}}, \widehat{P}_{\mathrm{SR}_{i}}} L\left(\lambda_{1}, \lambda_{2}, \widehat{P}_{S 1_{(i)}}, \widehat{P}_{S 2_{(i)}}, \widehat{P}_{\mathrm{SR}_{i}}\right),
$$

and the dual problem can be written as

$$
\min _{\lambda_{1}, \lambda_{2} \geq 0 \widehat{P}_{S 1_{(i)}}, \widehat{P}_{S 2_{(i)}}, \widehat{P}_{\mathrm{SR}_{i}}} L\left(\lambda_{1}, \lambda_{2}, \widehat{P}_{S 1_{(i)}}, \widehat{P}_{S 2_{(i)}}, \widehat{P}_{\mathrm{SR}_{i}}\right) .
$$

It is noteworthy that solving a dual problem is not always equivalent to solving the primal problem. It has been proven from duality theory that the optimal duality gap $d=D^{*}-f^{*} \geq 0$ always holds [25], where $D^{*}$ and $f^{*}$ denote the primal and dual optimal values, respectively. The optimal duality gap $d=0$ when the primal problem is convex. For the problem of our interest, the objective function (47) is concave, and constraints (48a) and (48b) are convex; therefore, the primal and dual problems have the same optimal solutions.

According to [26], the dual problem in (51) can be further decomposed into the following sequentially iterative sub-problems:

Sub-problem 1-power allocation: Given the dual variables $\lambda_{1}$ and $\lambda_{2}$, the optimal powers that maximize (50) can be obtained by solving

$$
\frac{\partial L}{\partial \widehat{P}_{S 1_{(i)}}}=0, \frac{\partial L}{\partial \widehat{P}_{S 2_{(i)}}}=0, \text { and } \frac{\partial L}{\partial \widehat{P}_{\mathrm{SR}_{i}}}=0 .
$$


After direct calculation and some simplification, we obtain the equivalent powers for $\operatorname{Sm}(m \in\{1,2\})$ and $\mathrm{SR}_{i}$ as

$$
\begin{aligned}
\widehat{P}_{S m_{(i)}}= & \sqrt[3]{-\frac{q_{m}}{2}+\sqrt{\left(\frac{q_{m}}{2}\right)^{2}+\left(\frac{p_{m}}{3}\right)^{3}}} \\
& +\sqrt[3]{-\frac{q_{m}}{2}-\sqrt{\left(\frac{q_{m}}{2}\right)^{2}+\left(\frac{p_{m}}{3}\right)^{3}}}, \\
\widehat{P}_{\mathrm{SR}_{i}}= & \left(\frac{1}{\lambda_{\mathrm{SR}_{i}}}\left(\sqrt{\frac{\lambda_{2}}{\lambda_{1}} g \lambda_{\mathrm{SR}_{i}}}-g\right)\right)^{+},
\end{aligned}
$$

where $(\cdot)^{+}=\max (\cdot, 0), u_{m}=\frac{1}{\alpha_{S m-S R_{i}}}+2 \frac{g}{\lambda_{S m}}-$ $\frac{1}{\lambda_{m} \beta_{m i}}, v_{m}=\left(\frac{g}{\lambda_{S m}}\right)^{2}+2 \frac{g}{\alpha_{S m-S R_{i}} \lambda_{S m}}+\frac{\left(\lambda_{\bar{m}}-2\right) g}{\lambda_{m} \beta_{m i} \lambda_{S m}}, w_{m}=$ $\left(\frac{1}{\alpha_{S m-S R_{i}}}-\frac{1}{\lambda_{m} \beta_{m i}}\right)\left(\frac{g}{\lambda_{S m}}\right)^{2}+\frac{\lambda_{\bar{m}}}{\lambda_{m}} \frac{1}{\alpha_{S m-S R_{i}} \beta_{m i}} \frac{g}{\lambda_{S m}}, p_{m}=v_{m}-$ $\frac{u_{m}^{2}}{3}, q_{m}=w_{m}+\frac{2 u_{m}^{3}}{27}-\frac{u_{m} v_{m}}{3}$, and $\bar{m}(\neq m) \in\{1,2\}$.

Sub-problem 2-dual variables update: To solve the minimization problem in (51), i.e., to find the optimal dual variables $\lambda_{1}$ and $\lambda_{2}$ for the given $\widehat{P}_{S 1_{(i)}}, \widehat{P}_{S 2_{(i)}}$, and $\widehat{P}_{\mathrm{SR}_{i}}$, a gradient-type search is guaranteed to converge to the global optimum, since the dual function is always convex [24]. Here, we use a subgradient update method. The basic idea of the subgradient method is to design a step-size sequence to update $\lambda_{1}$ and $\lambda_{2}$ in the subgradient direction. For the problem of our interest, the update may be performed as follows:

$$
\begin{aligned}
& \lambda_{1}(k+1)=\left(\lambda_{1}(k)-\xi_{1}(k) J_{1}\right)^{+}, \\
& \lambda_{2}(k+1)=\left(\lambda_{2}(k)-\xi_{2}(k) J_{2}\right)^{+},
\end{aligned}
$$

where $\xi_{1}$ and $\xi_{2}$ denote the update step sizes for $\lambda_{1}$ and $\lambda_{2}$, respectively, $k$ is the iteration index, and

$$
\begin{aligned}
& J_{1}=\widehat{P}_{\mathrm{SR}_{i}}-\beta_{1 i} \widehat{P}_{S 1_{(i)}}-\beta_{2 i} \widehat{P}_{S 2_{(i)}}, \\
& J_{2}=\frac{g}{\widehat{P}_{S 1_{(i)}} \lambda_{S 1}+g}+\frac{g}{\widehat{P}_{S 2_{(i)}} \lambda_{S 2}+g}+\frac{g}{\widehat{P}_{\mathrm{SR}_{i}} \lambda \mathrm{SR}_{i}+g}-3 \rho .
\end{aligned}
$$

Through the subgradient method, the powers for all relays can be allocated.

Once the power allocation is completed as described above, the relay selection is performed to maximize the sum achievable rate, i.e.,

$$
i^{\text {opt }}=\arg \max _{i} C_{S i \text { Sum }} \text {. }
$$

\subsection{Optimal power allocation scheme for data rate fairness (DRF)}

To guarantee the fairness between $S 1$ and $S 2$, the sum achievable rate with respect to the $i$ th relay can be denoted as

$$
C_{S_{i} \text {,Fair }}=2 * \min \left\{C_{S 1_{(i)}}, C_{S 2_{(i)}}\right\} .
$$

We will now determine the optimal equivalent power values $\widehat{P}_{S 1_{(i)}}, \widehat{P}_{S 2_{(i)}}, \widehat{P}_{\mathrm{SR}_{i,(1)}}$, and $P_{\mathrm{SR}_{i,(2)}}$ that lead to the fairness between $S 1$ and $S 2$, i.e., $C_{S 1_{(i)}}=C_{S 2_{(i)}}$ and then maximize the system achievable rate $C_{S_{i} \text {,Fair }}$.

In order to guarantee the primary QoS requirement, the constraint (9) should be satisfied with equality; otherwise, the achievable rate of SU can be further improved through increasing the powers at $S 1, S 2$, and $S R$. Also, the links of $S 1 \rightarrow S R, S R \rightarrow S 2, S 2 \rightarrow S R$, and $S R \rightarrow S 1$ should have the same SINR to guarantee fairness, i.e., $\alpha_{S 1-\mathrm{SR}_{i}} \widehat{P}_{S 1_{(i)}}=\alpha_{S 2-\mathrm{SR}_{i}} \widehat{P}_{S 2_{(i)}}=\alpha_{\mathrm{SR}_{i}-S 2} \widehat{P}_{S R_{i,(1)}}=$ $\alpha_{\mathrm{SR}_{i}-S 1} \widehat{P}_{S R_{i,(2)}}$. For simplicity, define $\frac{\alpha_{S 1-\mathrm{SR}_{i}}}{\alpha_{S 2-\mathrm{SR}_{i}}}=\alpha_{i}, \frac{\alpha_{S 1-\mathrm{SR}_{i}}}{\alpha_{S R_{i}-S 1}}=$ $\beta_{i}$, and $\frac{\alpha_{S 1-S R_{i}}}{\alpha_{S R_{i}-S 2}}=\gamma_{i}$, which equals to $\widehat{P}_{S 2_{(i)}}=\alpha_{i} \widehat{P}_{S 1_{(i)}}$, $\widehat{P}_{S R_{i,(2)}}=\beta_{i} \widehat{P}_{S 1_{(i)}}, \widehat{P}_{S R_{i, 1}}=\gamma_{i} \widehat{P}_{S 1_{(i)}}$, and $\widehat{P}_{S R_{i}}=\widehat{P}_{S R_{i,(2)}}+$ $\widehat{P}_{S R_{i,(1)}}=\left(\beta_{i}+\gamma_{i}\right) \widehat{P}_{S 1_{(i)}}$. By replacing $\widehat{P}_{S 2_{(i)}}$ and $\widehat{P}_{\mathrm{SR}_{i}}$ with $\widehat{P}_{S 1_{(i)}}$ into (9), the best power allocation for S1 should be satisfied with

$\frac{g}{\lambda_{S 1} \widehat{P}_{S 1_{(i)}}+g}+\frac{g}{\alpha_{i} \lambda_{S 2} \widehat{P}_{S 1_{(i)}}+g}+\frac{g}{\left(\beta_{i}+\gamma_{i}\right) \lambda_{S R_{i}} \widehat{P}_{S 1_{(i)}}+g}=3 \rho$.

Equation (59) is the univariate cubic equation about $\widehat{P}_{S 1_{(i)}}$ which can be solved by Cardano's formula. Once the best power allocation for $S 1$ is solved, the best power allocation for $S R$ and $S 2$ can also be achieved.

After the optimal power is allocated for all the relays, the relay which can maximize the sum achievable rate to guarantee the fairness is selected, i.e.,

$$
i^{\mathrm{opt}}=\arg \max _{i} C_{S_{i} \text {,Fair. }}
$$

\section{Simulation results}

In this section, we evaluate the performance of the proposed power allocation schemes through Monte-Carlo simulations and compare them with the traditional ERA schemes [18]. We assume throughout that the channel coefficients are i.i.d. and follow Rayleigh distribution. The following parameters are used throughout this section:

$\gamma=4, d_{\mathrm{PT}-\mathrm{PD}}=d_{S 1-\mathrm{SR}_{i}}=d_{S 2-\mathrm{SR}_{i}}=\frac{1}{2} d_{S 1-S 2}=1$, $d_{S 1-\mathrm{PD}}=d_{\mathrm{PT}-S 2}=4, d_{\mathrm{PT}-S 1}=d_{\mathrm{PT}-\mathrm{SR}}=d_{\mathrm{SR}-\mathrm{PD}}=$ $d_{S 2-\mathrm{PD}}=3, N_{0}=-50 \mathrm{dBW}$, and $R_{P}=1.5 \mathrm{bit} / \mathrm{s} / \mathrm{Hz}$.

Figure $2 \mathrm{a}-\mathrm{c}$ describes the $\mathrm{SU}$ achievable rate versus the PU transmit power $P_{\mathrm{PT}}$ with the PU outage threshold Pout ${ }_{\text {Pri,Thr }}=10^{-3}$ for the OWT-RA, TWT-DL, and TWT-RA networks, respectively. As can be seen from the figure, all the proposed power allocation schemes greatly outperform the traditional ERA schemes in the three different networks. It is also observed that there exists a cutoff point when $P_{\mathrm{PT}}$ changes. The reason is that the QoS of the PU will not be guaranteed when the power $P_{\mathrm{PT}}$ is too small, and thus, there will be no chance for the secondary transmissions. When $P_{\mathrm{PT}}$ is higher than the cutoff 


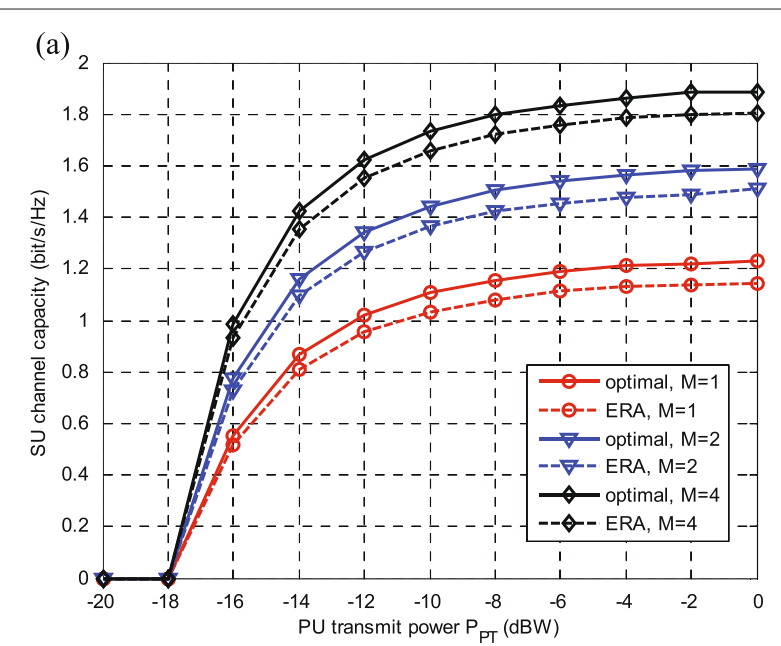

(b)
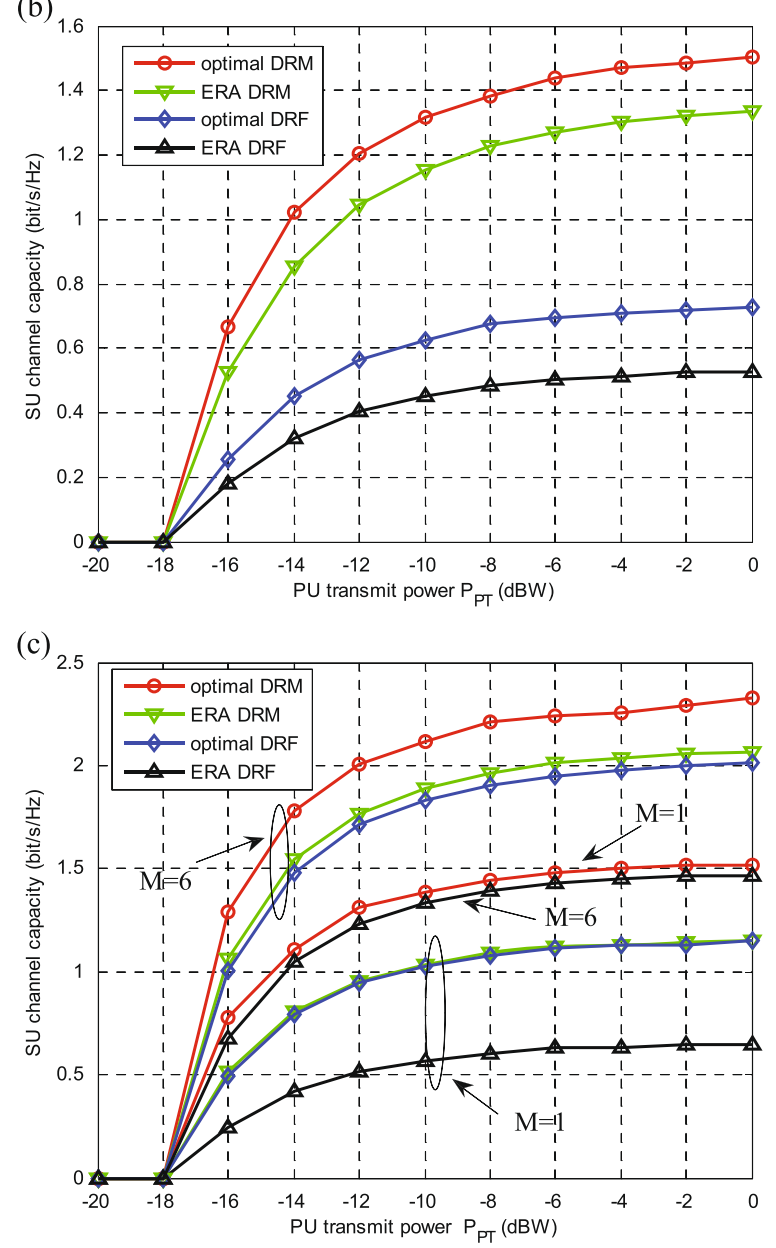

Fig. $2 \mathrm{SU}$ achievable rate versus $\mathrm{PU}$ transmit power $P_{\mathrm{PT}}$ for $\mathbf{a}$ the cognitive OWT-RA network, b the cognitive TWT-DL network, and c the cognitive TWT-RA network value, the SU transmission is enabled and the achievable rate of the SU improves with the increase of $P_{\mathrm{PT}}$. After $P_{\mathrm{PT}}$ increases further to a certain level, a performance ceiling is achieved. This phenomenon can be explained as follows: with the increase of $P_{\mathrm{PT}}$, more interference can be tolerated by the PU, hence, more transmission power can be allowed for the SU. However, when $P_{\mathrm{PT}}$ becomes large enough, the interference from the PU becomes the dominant factor which will affect the performance improvement of the SU. Besides, in the two-way transmission networks, such as the cognitive TWT-DL and TWT-RA networks, as shown in Fig. 2b, c, both the DRM and DRF schemes show the obvious improvement as compared to the corresponding ERA schemes. In addition, the DRF scheme tends to provide a lower achievable rate than the DRM scheme. That is because for the DRF scheme, to guarantee the fairness, the achievable rate of the $\mathrm{SU}$ is determined by the weaker link. As for the relay-assisted networks, such as the cognitive OWT-RA and TWT-RA networks, except for power allocation, the SU's achievable rate can be further improved by relay selection for more diversity gain is provided, as shown in Fig. 2a, c.

Figure $3 \mathrm{a}-\mathrm{c}$ describes the $\mathrm{SU}$ achievable rate versus the PU outage threshold Pout Pri,Thr with $P_{\mathrm{PT}}=0 \mathrm{dBW}$ for the three different networks as mentioned above. It is observed that all the proposed schemes provide better performance than the corresponding ERA schemes. With the increase of PoutPri,Thr, the SU's achievable rate can be improved for the reason that larger PoutPri,Thr implies the lower QoS requirement of the PU, which will allow for larger transmit power at SU. The performance difference among the proposed schemes and the corresponding ERA schemes becomes apparent with the increase of PoutPri,Thr, which verifies the effectiveness of our proposed schemes. Fig. 3b, c show the improvement of the proposed power allocation schemes in the twoway transmission networks (e.g., the cognitive TWT-DL and TWT-RA networks). Except for the power allocation, the effect of the relay selection is illustrated in Fig. 3a, c for the relay-assisted networks (e.g., the cognitive OWTRA and TWT-RA networks), which shows that with relay selection, the achievable rate of the SU can be greatly improved.

Figure $4 \mathrm{a}-\mathrm{c}$ describes the $\mathrm{SU}$ achievable rate versus the distance $d_{\mathrm{PT}-\mathrm{PD}}$ between PT and PD with PU outage threshold PoutPri,Thr $=10^{-3}$ and $P_{\mathrm{PT}}=0 \mathrm{dBW}$ for the three different networks as mentioned above. We can see from the figure that all the proposed power allocation schemes provide better performance than the corresponding ERA schemes. When the primary link from $\mathrm{PT}$ to $\mathrm{PD}$ is in poor condition, i.e., $d_{\mathrm{PT}-\mathrm{PD}}$ is large, the primary QoS requirement can hardly be guaranteed; therefore, SU must suppress its powers to protect PU and degrade SU's performance as a result. 


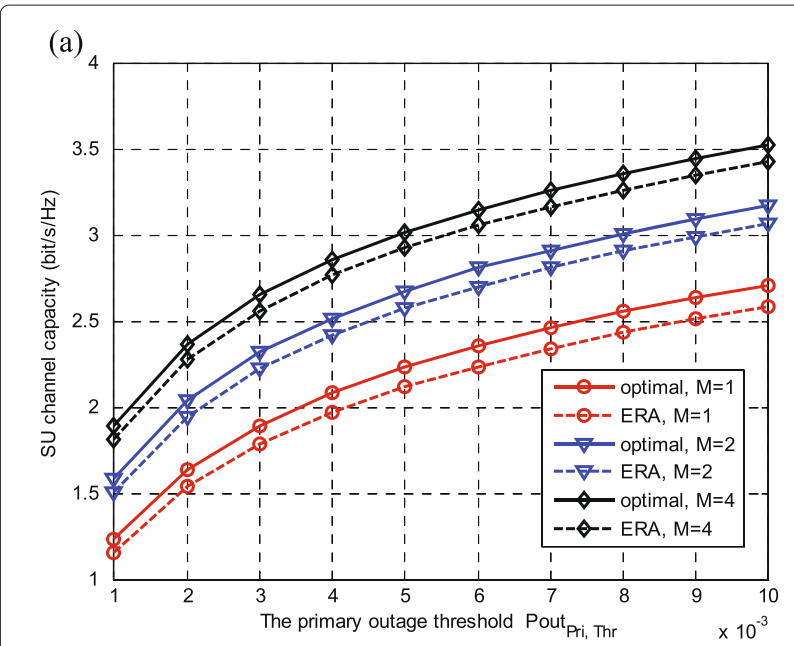

(b)
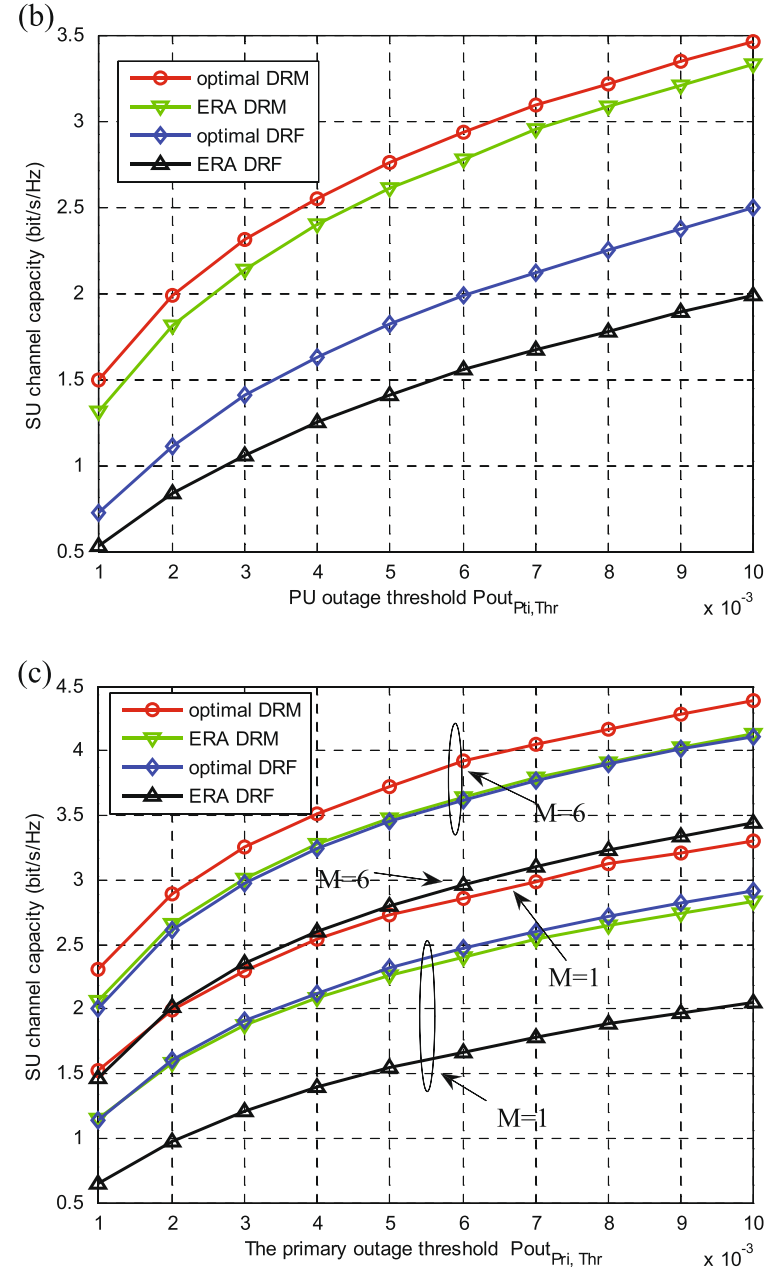

Fig. $3 \mathrm{SU}$ achievable rate versus PU outage threshold PoutPri,Thr for a the cognitive OWT-RA network, b the cognitive TWT-DL network, and $\mathbf{c}$ the cognitive TWT-RA network

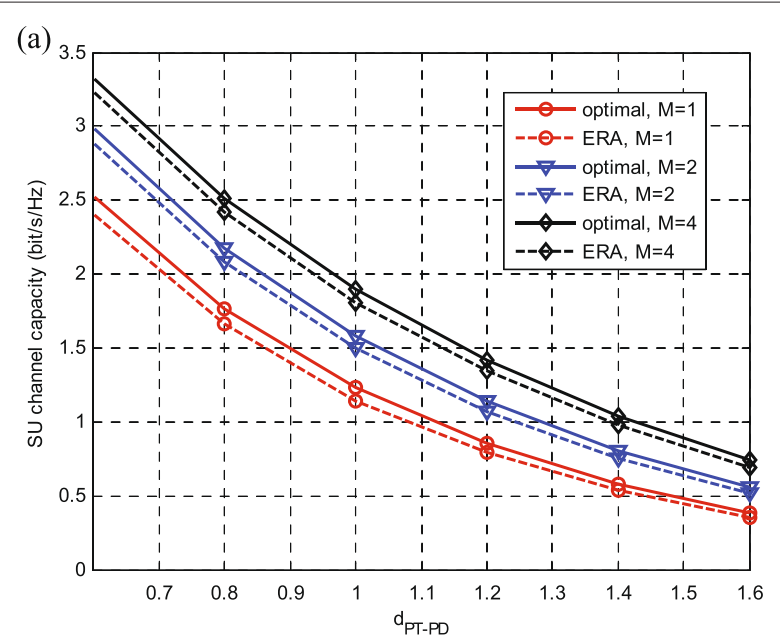

(b)

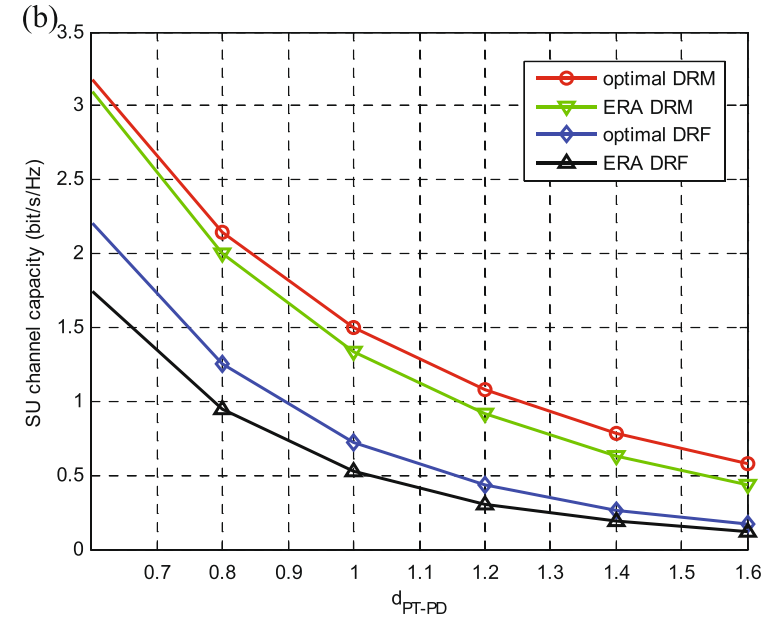

(c) 4

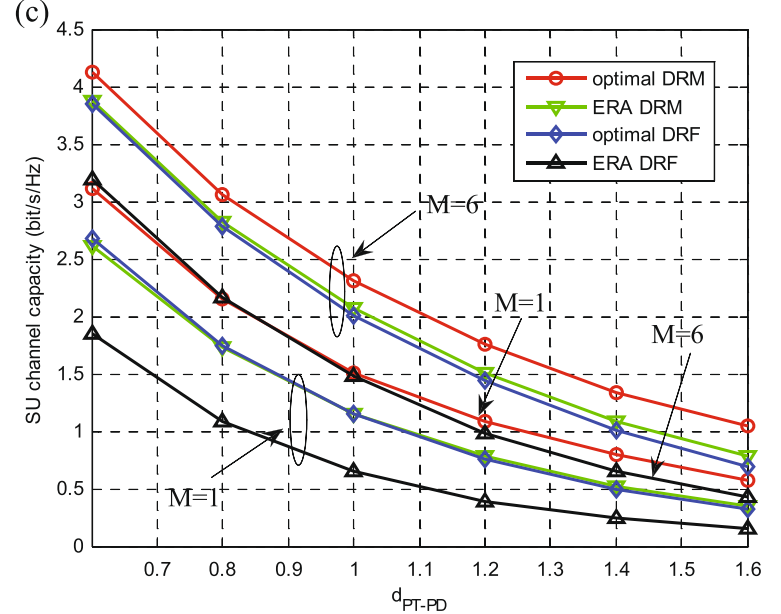

Fig. 4 SU achievable rate versus PU parameter $\sigma_{\text {PT_PD }}^{2}$ for a the cognitive OWT-RA network, $\mathbf{b}$ the cognitive TWT-DL network, and c the cognitive TWT-RA network 
With the decrease of $d_{\mathrm{PT}-\mathrm{PD}}$, the SU's performance can be improved accordingly. The performance difference among the proposed schemes and the corresponding ERA schemes becomes apparent with the decrease of $d_{\text {PT-PD, which verifies the effectiveness of our proposed }}$ schemes. In addition to power allocation, the effect of the relay selection is illustrated in Fig. 4a, c, manifesting the improvement of SU's performance through relay selection.

\section{Conclusions}

In this article, we studied the problem of power allocation for underlay cognitive networks involving three different secondary transmission models: (i) OWT-RA, (ii) TWT-DL, and (iii) TWT-RA, respectively. Since secondary network is typically not coordinated with primary network, the instantaneous CSI of PU can hardly be obtained. To tackle this issue, we adopted primary outage constraint to measure the QoS of primary transmission, where only the statistical CSI of PU required. We first derived the joint primary outage constraints due to the secondary transmission for the three transmission models. We then proposed power allocation schemes to maximize the received SINR for the cognitive one-way relay network, while in cognitive twoway scenarios, two power allocation criterions are considered: (i) fairness based and (ii) sum achievable rate maximization based, respectively. In order to further improve the SU's performance, relay selection was also considered in the relay-assisted transmission networks (e.g., OWT-RA and TWT-RA networks). The performance of the proposed schemes was illustrated for different operating conditions and shown to yield great enhancement compared to that of the corresponding ERA schemes.

\section{Competing interests}

The authors declare that they have no competing interests.

\section{Authors' contributions}

$\mathrm{PL}$ contributed in the conception of the study and design of the study and wrote the manuscript. FS carried out the simulation and revised the manuscript. LC participated in the design of the study. GZ helped perform the analysis with constructive discussions and helped to draft the manuscript. All authors read and approved the final manuscript.

\section{Acknowledgements}

This work was supported in part by the Shandong Province High School Science \& Technology Fund Planning Project (J12LN02) and the Research Fund for the Doctoral Program of Higher Education of China (20123702120016).

\footnotetext{
Author details

${ }^{1}$ College of Information Science and Engineering, Shandong Agricultural University, Daizong Road No.61, 271018 Tai'an, Shandong, China. ${ }^{2}$ Administration Center of Shandong Academy of Information \& Communication Technology, Xinluo Street No. 1768, High-tech Zone, 250101 Jinan, China.
}

Received: 15 September 2015 Accepted: 16 October 2015 Published online: 31 October 2015

\section{References}

1. Q Zhao, BM Sadler, A survey of dynamic spectrum access. IEEE Signal Process Mag. 24(3), 79-89 (2007)

2. S Haykin, Cognitive radio: brain-empowered wireless communications. IEEE J. Select. Areas. Commun. 23(2), 201-220 (2005)

3. M Gastpar, M Vetterli, in On the capacity of wireless networks: The relay case. INFOCOM 2002. Twenty-First Annual Joint Conference of the IEEE Computer and Communications Societies. Proceedings. IEEE, vol. 3 (IEEE New York, 2002), pp. 1577-1586

4. G Kramer, M Gastpar, P Gupta, Cooperative strategies and capacity theorems for relay networks. IEEE Trans. Inf. Theory. 51(9), 3037-3063 (2005)

5. Q Zhang, J Jia, J Zhang, Cooperative relay to improve diversity in Cognitive radio networks. IEEE Commun. Mag. 47(2), 111-117 (2009)

6. B Rankov, A Wittneben, Spectral efficient protocols for half-duplex fading relay channels. IEEE J. Select. Areas. Commun. 25(2), 379-389 (2007)

7. R Zhang, Y-C Liang, CC Chai, S Cui, Optimal beamforming for two-way multi-antenna relay channel with analogue network coding. IEEE J. Select. Areas. Commun. 27(5), 699-712 (2009)

8. C Sun, C Yang, Energy efficiency analysis of one-way and two-way relay systems. EURASIP J. Wirel. Commun. Netw. 2012(1), 1-18 (2012)

9. A Ghasemi, ES Sousa, Fundamental limits of spectrum-sharing in fading environments. IEEE Trans. Wirel. Commun. 6(2), 649-658 (2007)

10. A Alsharoa, F Bader, M-S Alouini, Relay selection and resource allocation for two-way df-af cognitive radio networks. IEEE Wirel. Commun. Lett. 2(4), 427-430 (2013)

11. X Kang, Y-C Liang, A Nallanathan, HK Garg, R Zhang, Optimal power allocation for fading channels in cognitive radio networks: Ergodic capacity and outage capacity. IEEE Trans. Wirel. Commun. 8(2), 940-950 (2009)

12. X Kang, R Zhang, Y-C Liang, HK Garg, Optimal power allocation strategies for fading cognitive radio channels with primary user outage constraint. IEEE J. Select. Areas Commun. 29(2), 374-383 (2011)

13. L Li, X Zhou, H Xu, GY Li, D Wang, A Soong, Simplified relay selection and power allocation in cooperative cognitive radio systems. IEEE Trans. Wirel. Commun. 10(1), 33-36 (2011)

14. L Lu, Y Li, G Wu, Optimal power allocation for cr networks with direct and relay-aided transmissions. IEEE Trans. Wirel. Commun. 12(4), 1-11 (2013)

15. M Naeem, U Pareek, DC Lee, A Anpalagan, Power allocation and relay assignment for shared-band nonregenerative relaying in cognitive radio systems. IEEE Trans. Veh. Technol. 62(6), 2853-2859 (2013)

16. X Kang, Optimal power allocation for bi-directional cognitive radio networks with fading channels. IEEE Wirel. Commun. Lett. 2(5), 567-570 (2013)

17. P Ubaidulla, S Aissa, Optimal relay selection and power allocation for cognitive two-way relaying networks. IEEE Wirel. Commun. Lett. 1(3), 225-228 (2012)

18. Y Zou, J Zhu, B Zheng, Y-D Yao, An adaptive cooperation diversity scheme with best-relay selection in cognitive radio networks. IEEE Trans Signal Process. 58(10), 5438-5445 (2010)

19. SB Mafra, RD Souza, JL Rebelatto, EM Fernandez, H Alves, Cooperative overlay secondary transmissions exploiting primary retransmissions. EURASIP J. Wirel. Commun. Netw. 2013(1), 1-12 (2013)

20. $\mathrm{P}$ Lan, $\mathrm{F}$ Sun, $\mathrm{L}$ Chen, $\mathrm{P}$ Xue, J Hou, Power allocation and relay selection for cognitive relay networks with primary qos constraint. IEEE Wirel. Commun. Lett. 2(6), 583-586 (2013)

21. I Krikidis, J Thompson, JS McLaughlin, N Goertz, Amplify-and-forward with partial relay selection. IEEE Commun. Letters. 12(4), 235-237 (2008)

22. DB Costa, S Aissa, Performance analysis of relay selection techniques with clustered fixed-gain relays. IEEE Signal Proc. Lett. 17(2), 201-204 (2010)

23. J-p Hong, B Hong, TW Ban, W Choi, On the cooperative diversity gain in underlay cognitive radio systems. IEEE Trans. Commun. 60(1), 209-219 (2012)

24. S Boyd, L Vandenberghe, Convex Optimization. (Cambridge university press, Cambridge, 2009)

25. DT Ngo, C Tellambura, HH Nguyen, Resource allocation for ofdma-based cognitive radio multicast networks with primary user activity consideration. IEEE Trans. Veh. Technol. 59(4), 1668-1679 (2010)

26. AG Marques, X Wang, GB Giannakis, Dynamic resource management for cognitive radios using limited-rate feedback. IEEE Trans. Signal Process. 57(9), 3651-3666 (2009) 